\title{
Curcumin: New Weapon against Cancer
}

\author{
Fayez Hamam \\ Department of Pharmacology and Toxicology, College of Pharmacy, Taif University, Taif, KSA \\ Email: f.hamam@tu.edu.sa
}

Received 22 September 2014; revised 13 November 2014; accepted 2 December 2014

Copyright (C 2014 by author and Scientific Research Publishing Inc.

This work is licensed under the Creative Commons Attribution International License (CC BY). http://creativecommons.org/licenses/by/4.0/

(c) () Open Access

\section{Abstract}

All the evidences point out to the fact that the incidence, mortality and number of persons living with cancer are on the rise and, thus, this will impose a significant burden on health care resources. The considerable number of deaths from cancer necessitates the need to developing novel alternative cures that are efficient, safe, cheap and easy to use. In the search for new therapies for tumors, naturally-derived compounds have been considered as a good source of novel anticancer drugs. The challenge here is to find products that are pharmacologically active against tumor cells with suitable toxicity profile and least damage to normal cells. Curcumin is a spice widely used in many countries especially in South Asia and it has gained importance for its anticancer function and low toxicity toward normal tissues in a range of biological systems. In spite of significant research works, many difficulties hinder its oral use in the therapy of different kind of tumors, such as extreme low solubility in water, quick break down and excretion after being absorbed in the human body. Low bioavailability due to enhanced metabolism and rapid system elimination is another problem that hinders oral use of curcumin as anticancer agent. Therefore, the previously mentioned poor pharmacokinetics characteristics inhibit curcumin from reaching its site of action and, thus, lessen its effectiveness against tumors. This article reviews the latest global cancer statistics with special attention to be directed toward ovarian cancer. It sheds light on many research works that investigated the protective and therapeutic functions of different curcumin preparations against different sites of cancer using animal models. It also summarizes recent research works concerning the antitumor effects of curcumin alone and/or loaded into a range of delivery devices in many types of ovarian cancer cell lines. In the last section, it discusses the latest research work with regard ovarian cancer in the kingdom of Saudi Arabia.

\section{Keywords}

Curcumin, Cancers, Ovarian Cancers, Delivery Systems, In Vitro, In Vivo 


\section{Cancer}

\subsection{A great Threat to Human}

In the year 2012, world cancer statistics estimated cancer incidence (14.1 million) and mortality (8.2 million) worldwide. The number of new cases could further rise to 19.3 million by the year 2025, possibly due to growth and aging of the world population [1]. According to the International Agency for Research on Cancer, the most widely diagnosed tumors globally were lung (13.0\%), breast (11.9\%) and colorectum (9.7\%) [2], while death from lung cancer was ranked first (19.1\%) followed by liver cancer (9.1\%), stomach cancer accounted for 8.8\% of total deaths from cancers [2]. In economically developed countries, deaths from cancer are the leading cause of mortality. In the meantime, it is the second leading cause of deaths in developing countries. The load of cancer is rising in economically developing world as a result of several reasons, including growth and aging of population, an adoption of cancer-related lifestyle such as smoking, physical immobility, and non-healthy "westernized" foods [3].

\subsection{Ovarian Cancer}

In 2002, it was estimated that $19 \%$ of the 5.1 million new cases of worldwide women had various kinds of gynaecological cancers. While the mortality of women suffered gynaecological cancers was 2.9 million and the number of deaths could be increased to 13 million in a 5-year prevalent tumor cases. Incidence and mortality of ovarian cancer were 204,000 and 125,000, respectively [1]. Although ovarian cancer is ranked the sixth among most common tumors in the globe, it is the leading cause of mortality among gynecologic malignant tumors and, thus, it is selected as the main topic of the present review.

\subsection{Current Status of Ovarian Cancers in the Kingdom of Saudi Arabia}

In 2010, ovarian tumor was ranked as the fifth most common tumor diagnosed in women in KSA [4]. In a retrospective study conducted at King Faisal Specialist Hospital and Research Center between 1995 and 2007, 193 female patients with epithelial form of ovarian cancer were identified and results showed that $67.4 \%$ had stage III disease and $20.2 \%$ had stage IV disease [4]. The percent of woman with advanced-stage of ovarian cancer is higher among Saudi women compared to their counterparts worldwide. Furthermore, the researchers found that $85 \%$ of women with this kind of ovarian cancer had no family history of ovarian tumor [4]. Of 1000 consecutive malignant carcinoma among Saudi residents in the Western part of the KSA, cervical cancer (42 cases) was the most common, followed by uterine cancer ( 24 cases) and ovarian cancer (21 cases) [5]. In order to study the effect of foreign immigration between 1985 and 2007 on the incidence of gynecological cancers, a retrospective study of histopathological records was conducted. Results of this study showed that there was no difference between foreign immigrants and Saudi nationals in number of new cases of uterine corpus sarcoma, ovarian and vaginal cancer. Moreover, incidence of cancers of cervix, vulva, uterine and gestational trophoblastic neoplasia altered over time [6]. Sait [7] conducted a retrospective study of 39 female patients treated traditionally for ovarian tumor between 2000 and 2010 at King Abdulaziz University Hospital in the city of Jeddah, KSA. He found that $80 \%$ of patients had stage I cancer and $20 \%$ had Germ cell cancer. He also showed that $98 \%$ of affected women returned their menstrual cycle after conservative treatment and $20 \%$ of patients became pregnant. Similarly, Al-Badawi and his fellows [8] found that bilateral laparoscopic ovarian transposition performed in 23 premenopausal females with pelvic malignancy was safe and efficient method for restoring ovarian function. In another clinical study conducted by Al-Badawi and his colleagues [9] to assess postoperative chemotherapy in 55 patients with advanced ovarian cancer (stage III and IV), the authors found that $60 \%$ of them after primary surgical intervention recurred the disease within 4.5 years from first diagnosis. Furthermore, patients had gross residual tumor more than $2 \mathrm{~cm}$ died within 4 years of diagnosis. The researchers concluded that the presence of macroscopic residual tumor following first surgery was the main crucial prognostic variable [9]. It is clear from previously reviewed articles that the main focus of researchers in the KSA was toward clinical studies of ovarian cancer.

\section{Role of Curcumin in Inhibition of Cancer}

\subsection{Curcumin}

Curcumin [1,7-bis (4-hydroxy-3-methoxyphenyl)-1,6-heptadiene-3,5-dione], is a spice yellow in nature and ex- 
tracted from the roots of a member of ginger family (Curcuma longa). It is widely used in many countries particularly in South Asia and it has attracted attention for its anticancer role and decreased toxicity toward normal cells in several biological systems.

\subsection{Anticancer Effects of Curcumin Using Animal Models}

Over the past five decades considerable research studies have shown protective and therapeutic roles of curcumin against many types of cancers. The anticancer activities of curcumin (a polyphenol spice derived from the plant Curcuma longa, usually known as turmeric) have been investigated in several animal cancer models [4]-[19]. Whilst curcumin has good anti-cancer effect in vitro, when it is given in vivo, it fails to exhibit such anticancer effects due to its poor pharmacokinetic characteristics. Thus, several research works have developed a range of delivery systems for this pharmacologically active compound. For example, Chen and his coworkers [14] prepared three liposomal preparations composed of three different types of phospholipids. Among the three tested preparations, curcumin encapsulated into soybean phospholipids liposome exhibited a marked antimelonoma effect in C57BL/6 mice in comparison to the control group (curcumin alone solution). Huang et al. [4] chemically initiated cancers on skin of female CD-1 mouse via 2,4-Dimethoxybenzaldehyde (DMBA), while induction of cancer was conducted using 12-O-tetradecanoylphorbol-13-acetate (TPA). A considerable inhibition of skin cancers and epidermal synthesis of DNA was noted upon topical application of curcumin dissolved in acetone over a 20-week study period. However, this study neither explored in vitro skin permeation of curcumin alone (unloaded) nor tried to develop a suitable delivery device for this highly lipophilic compound (curcumin) [4]. The same research team [10] employed the same procedure for chemical promotion of skin cancer in CD1 female mice. Results of this study indicated that topical application of a minimal dose of unloaded curcumin and TPA resulted in a significant suppression of skin cancer growth. A similar study was conducted and used the same protocol for chemical promotion of skin cancer (Tumor initiation using DMBA, while induction using TPA). Different kinds of curcumin were evaluated for their possible inhibitory role on promotion of cancer using CD-1 mice. Commercial curcumin, pure curcumin, demethoxycurcumin, bisdemethoxycurcumin and tetrahydrocurcumin were applied topically. Results of this study showed that the first three kinds of curcumin (commercial curcumin, pure curcumin, and demethoxycurcumin) exhibited the same inhibition effects on cancer growth [6]. The previously summarized research works showed that topically applied curcumin as such or curcumin loaded into delivery systems (e.g. liposomes) had certain extent of inhibition on skin cancer promotion. Nonetheless, the previously mentioned works did not explored mechanism of action of curcumin as a potential anticancer agent. For instance, the investigators did not explored whether curcumin have an effect on modulating signaling pathways, such as its effect in suppression of nuclear transcription factor ${ }_{K} B\left(\mathrm{NF}_{K} \mathrm{~B}\right)$ or modulation of cytokines or stimulation of apoptosis (programmed cell death).

\subsection{Effects of Unloaded Curcumin (Free) Ovarian Cancer Growth in Vitro}

Liduan et al. [16] examined inhibitory effects of unloaded curumin on growth of ovarian tumor cells (A2780) using the the 3-(4,5-dimethylthiazol-2-yl)-2,5-diphenyltetrazolium bromide (MTT) assay and results showed that curcumin suppressed the growth of these cancer cells in a time- and dose-dependent manner. Inversion microscope indicated certain morphological changes (irregular shape with a high number of round cells) of ovarian cells. The rates of cell death following treatment with curcumin were $6.41 \%-28.48 \%$. Molecular mechanism study was conducted using the immunohistochemistry method and results indicated that the expression of proteins (bcl-2 and P53) was decreased in a dose- and time-dependent manner. The same research group investigated the potential role of curcumin on growth inhibition inducing mechanisms in human ovarian cells (A2780). Results showed the inhibition rates of tumor cells were $62.05 \%-89.24 \%$ using the MTT assay and the half inhibitory concentration $\left(\mathrm{IC}_{50}\right)$ of curcumin was determined $(\sim 47 \mu \mathrm{mol})$ after 12 hours of incubation of curcumin and A2780 ovarian cancer cells. Furthermore, curcumin induced apoptosis of ovarian tumor cells in vitro using the fragmentation of cellular DNA, and this apoptosis stimulating effect of curcumin was further studied using the transmission electron microscope which indicated certain ultra-structural changes [15]. Investigating of immunohistochemistry of the proteins, nuclear factor-Kappa B (NF-K $\mathrm{B}, \mathrm{P} 65)$ and cysteinyl aspartate specific protease-3 (Caspase-3), indicated that the expression of $\mathrm{NF}_{-\mathrm{K}} \mathrm{B}$ (P65) was lowered, while the expression of Caspase-3 was increased in a dose-dependent manner [15]. Similarly, Shi et al. [17] used different kind of human ovarian cancer cells ( Ho-8910) and results showed that at a dose of $40 \mu \mathrm{M}$ of curcumin and 48 hours of expo- 
sure to curcumin, the rate of growth suppression reached 52\% using the MTT assay. Molecular mechanism study using flow cytometry and Western blotting revealed a decline in the expression of proteins implicated in the process of apoptosis, namely Bcl-2, Bcl-XL (important regulatory molecules of cell death) and pro-caspase-3. On the other hand, the amount of cancer inhibitor protein (p53) was considerably increased. This protein play a central role in the regulation of apoptosis and it is believed that its expression is increased in response to DNA damage [17]. Although the previously mentioned research works indicated potential antitumor effects of curcumin and hinted about possible molecular mechanism of this drug, none of these studies tried to address many obstacles that hinder the oral use of curcumin as a pharmacological active compound in treatment of different sites of cancers, such as its extreme low solubility in water ( $\sim 11 \mathrm{ng} / \mathrm{ml})$ [20] [21], rapid degradation and excretion following absorption in the human body [22] [23], and it has low bioavailability due to its quick metabolism and fast system elimination.

\subsection{Evaluation of the Antitumor Potential of Curcumin Loaded into Different Delivery Systems in Vitro}

The antitumor effects of curcumin have been characterized in many cell culture models [6] [12] [14]-[17] [24]-[28]. For example, Dhule et al. [14] developed a novel delivery system for curcumin which encapsulated curcumin into cyclodextrin then packaging the complex in a liposome. The resulting curcumin nanoformulation showed promising results in vitro against KHOS osteosarcoma cell line and MCF-7 breast cancer cell line. Furthermore, the researchers found that curcumin-loaded cyclodextrin liposomal nanoparticles initiated the caspase cascade which leads to apoptotic cell death which is preferred over autophagy as it yields least level of immune response following cell death [12]. Chen et al. [14] used different kinds of phospholipids for the preparation of three different curcumin-loaded liposomes. The in vitro antitumor activity of the three liposomes was investigated against B16BL6 melanoma cells in an MTT assay. Among the three preparations tested, curcumin-soybean phospholipids-liposome displayed the highest capacity of all loaded liposomes to inhibit the growth of B16BL6 melanoma cells. However, this study did not investigate mechanism(s) or mode of action of curcumin loaded liposomes as antitumor agents.

The cytotoxicity of the curcumin loaded chitin nanogels (CCNGs) was investigated on human dermal fibroblast cells (HDF) and human melanoma (A375) cell lines. The MTT test showed that the resultant nanogels displayed specific toxicity towards A375 melanoma cells, but less toxicity towards the control human dermal fibroblast cells. The confocal analysis confirmed the intake of curcumin loaded chitin nanogels by melanoma cell lines. The CCNGs induced apoptosis using a flow-cytometric assay. Curcumin loaded chitin nanogels has an effect on the two major apoptotic pathways: the mitochondrial (intrinsic) and death receptor (extrinsic) [11].

Two different cell lines (CAL27 and UM-SCC1), both representing head and neck squamous cell carcinoma (aggressive form of oral cancers) were examined for growth suppression with different doses of liposomal curcumin and results indicated a considerable suppression of growth using liposomal curcumin compared to unloaded liposome. Inhibition of growth of CAL27 treated with liposomal curcumin was dose-dependent. Furthermore, this study investigated the mechanism of growth inhibition using a reporter gene assay and found that liposomal curcumin inhibited the activation of the nuclear factor ${ }_{k} \mathrm{~B}\left(\mathrm{NF}_{\mathrm{k}} \mathrm{B}\right)$ which is an inducible transcription element implicated in the pathogenesis of many malignancies including skin cancers [15].

Li et al. [25] found that exposure of 6 cell lines from human pancreatic carcinoma inhibited cell proliferation in a dose and time-related manner using MTT assay. Annexin V/Propidium Iodide Staining Assay showed that liposomal curcumin increased apoptosis (programmed cell death) in a dose-dependent manner and the effects were equivalent to or higher than those of free curcumin at equimolar concentrations. Electrophoretic Mobility Shift Assay (EMSA) indicated that $\mathrm{NF}_{\mathrm{k}} \mathrm{B}$ activation was inhibited upon treatment of all six cell lines with liposomal curcumin in a dose-related manner. Moreover, liposomal curcumin decreased the steady-state amount of $\mathrm{NF}_{k} \mathrm{~B}-$ regulated gene Products (Interleukin-8; IL-8 and COX-2) using immunoblotting assay. These two molecules are implicated in the growth and metastatic potential of this kind of tumors [7].

Huang et al. [26] compared the pharmacological effects of four kinds of curcumin: commercial food grade curcumin, pure curcumin, demethoxycurcumin, bisdemethoxycurcumin and tetrahydrocurcumin using cultured JB6 $(\mathrm{P}+)$ cells. The results showed that the first three kinds were equally potent, while tetrahydrocurcumin was less active in terms of inhibition of TPA-induced tumor growth. Nonetheless, these studies did not investigate mode of action of curcumin as anticarciongens or whether these biologically active curcumin affect apoptosis, 
signaling pathways and angiogenesis [6].

\subsection{Inhibition Effects of Loaded Curcumin on Growth of Ovarian Cancer in Vitro}

Ganata and Amiji [27] encapsulated curcumin and the drug paclitaxel in flaxseed oil contained nanoemulsion formulations. Results showed coadmistration of the drugs, curcumin and paclitaxel, were efficiently delivered inside cells in both sensitive ovarian cancer (SKOV3) and drug resistant (SKOV3 ${ }_{\mathrm{TR}}$ ) cells. Mechanism of anti-cancer effect of this nanoemulsion indicated that curcumin inhibited activity of nuclear factor kappa $\mathrm{B}\left(\mathrm{NF}_{\mathrm{k}} \mathrm{B}\right)$ as well as expression of P-glycoprotein drug resistant cells. Furthermore, coadmistration of nanoemulsion of curcumin and paclitaxel was very toxic against both types of cells by enhancing apoptosis. Kumar et al. [28] loaded curcumin into the core of hydrophilic polymeric [poly(2-hydroxyethyl methacrylate); PHEMA] nanoparticles. PHEMA gels were made using choline based liquid such as choline formate. MTT test indicated that curcumin loaded PHEMA nanoparticles displayed higher level of cytotoxicity than free curcumin against drug sensitive human ovarian cancer cells (SKOV-3). The study also showed that the $\mathrm{IC}_{50}$ for loaded curcumin PHEMA nanoparticles was lower $(3.78 \mu \mathrm{g} / \mathrm{ml})$ than that of unloaded curcumin $(17.25 \mu \mathrm{g} / \mathrm{ml})$. Western blotting study showed significant change in concentration of proteins directly linked to cell death (apoptosis). For example, loaded curcumin inhibited function and expression of nuclear factor kappa $\mathrm{B}\left(\mathrm{NF}_{\mathrm{k}} \mathrm{B}\right)$ and, hence, limit the cancer cell lines. Curcumin-PHEMA-nanoparticles triggered programmed cell death in tumor cells and it was evident from the decreased expression of antiapoptotic Survivin (important regulatory protein for apoptosis ) and decreased expression of VEGF (a stimulator of endothelial cell growth in many human cancer sites) and COX-2 (important in disease progress) [28]. Wahl et al. [19] used two kinds of chemo resistant ovarian cell lines (SKOV-3 and ES-2) to investigate whether curcumin could trigger cell death in ovarian cancer cells and improve programmed cell death induced by cancer necrosis factor-related apoptosis enhancing Apo 2 ligand/ TRAIL (known as Apo 2L/TRAIL). Sulforhodamine test indicated that free curcumin was toxic against cisplatin-resistant cancer cells at a dose of $25 \mu \mathrm{M}$. The combined treatment of curcumin and Apo 2L/TRAIL activated both the intrinsic pathway (mitochondria-mediated cell death pathway, as indicated by the cleavage of caspase-9 protein) and the extrinsic pathway (receptor-mediated cell death) which is characterized by the stimulation of the death receptors (DR4 and DR5) on the cell surface and it was indicated through the cleavage of caspase-8 protein. Therefore, combination of curcumin and Apo 2L/TRAIL treatment could bypass obstacle of chemo resistant of ovarian cancer to the standard chemotherapeutics drugs [19]. In a similar study conducted by Yunos and colleagues [18] to determine the synergetic inhibitory effects of curcumin with cisplatin and a phytochemical (Epigallocatechin-3-gallate) on growth of human ovarian cell lines (cisplatin-sensitive A2780 and cisplatin resistant $\mathrm{A} 2780^{\mathrm{cisR}}$ ). Levels of platinum accumulated in the cells and linked to the DNA were measured using graphite furnace atomic absorption spectrometry. Results of this study showed that treatment of cell lines (A2780 and A2780 ${ }^{\text {cisR }}$ ) with cisplatin 4 hours before addition of curcumin and the phytochemical resulted in the most synergistic effects. Another research group [19] examined the combined effects of a curcumin analog (known as HO-3867) and cisplatin on proliferation of cisplatin resistant ovarian cancer cell lines $\left(\mathrm{A} 2780^{\mathrm{R}}\right)$. Cell viability test (MTT) indicated that combination of HO-3867 and cisplatin significantly suppressed growth of cisplatin-resistant cancer cells and this inhibition was in a dose-related manner. Western blotting and cell cycle analysis (FACS) showed that the growth inhibition was linked with elevated level of expression of regulatory proteins (p53 and p21). Meanwhile, these tests indicated that the expression of cdk5 and cyclin D1 was reduced. Immunoblotting analysis showed that the cell death was linked with the modulation of Bcl-2 family of proteins, specifically Bcl-2, Bax and Bcl-xL. One main findings of this study was decreased expression of cleaved caspase-3, -7 and PARP in treatment groups exposed to the combination of HO-3867 and cisplatin, thus suggesting that combined treatment might induce the signal transducer and activator of transcription-3 (STAT3) in the tested cell lines. STAT3 is known as a tumor transcription factor which has attracted immense significance as a pharmacologic target [19].

\subsection{Possible Mechanism of Actions of Curcumin as Antitumor Agent in Vivo}

The following studies investigated mechanism of action of curcumin as antitumor agent in vivo. Dhule et al. [12] used curcumin-encapsulated in $\gamma$-cyclodextrin followed by second loading in liposomes as delivery vehicles for treatment of osteosarcoma. This novel route for delivering curcumin displayed promising anticancer effect in immune deficient nude mice injected with KHOS cells [12]. Nude mice bearing tumours from the head and neck 
squamous cell carcinoma cell line (CAL2) were treated with liposome encapsulated curcumin. Liposomal curcumin showed significant xenograft tumor growth suppression compared with control mice and mice treated with unloaded liposomes. Immunohistochemistry study indicated mechanism of growth suppression of liposomal curcumin via the inhibition of transcription factor $\left(\mathrm{NF}_{\mathrm{k}} \mathrm{B}\right)$ [28]. It should be noted that this study has administered liposomal curcumin via an invasive route (i.v.). The human pancreatic carcinoma cell lines (BxPC-3 or MiaPaCa2 cells) were injected onto female nude mice then liposomal curcumin was administered after tumor masses became established. Substantial tumor growth suppression without obvious host toxicity was noted. Furthermore, tumors treated with liposomal curcumin showed an antiangiogenic effect (process of formation of new blood vessels which is vital for growth and development and it plays critical role in the transition of benign tumors to malignant), including apparent blanching of tumors upon visual inspection and decreasing expression of an endothelial marker(CD31) and VEGF and IL-8. It is noteworthy that pancreatic carcinoma cells usually express increased levels of IL-8 and cyclooxygenase-2 (COX-2), and these factors play a role in the growth and metastatic potential of this caner [7]. A recent study that was conducted by Al Badawi and his coworkers [8] showed that a single dose of curcumin- micro particles composed of poly(D, L-lactide-co-glycolide) polymer (PLGA) into BALB/c female mice could sustain levels of curcumin in the blood for a month and, hence, efficiently suppress tumor growth in a mouse model of breast tumor [8]. Ghosh et al. [12] prepared small particle size (diameter $\sim 15 \mathrm{~nm}$ ) of curcumin encapsulated into polylactide co-glycolide (PLGA). Hepatocellular carcinoma was induced using diethylnitrosamine (DEN) in male Swiss Albino rats. DEN resulted in the production of reactive oxygen species (ROS), lipid oxidation, and a decline in plasma membrane micro viscosity and a reduction of antioxidant enzyme levels in liver cells. Nano Cur administered orally in DEN induced HCC rats showed substantial protection against hepatocellular carcinoma. For example, Nano Cur decreased ROS in mitochondria and showed a noteworthy protection from increase of conjugated dienes (primary lipid oxidation product) in the liver cell membrane. Furthermore, Nano Cur treated rats showed significant reduction in important marker enzymes of liver. Results of this study indicated that this Nano Cur can act as antioxidant by preventing production of high amounts of ROS in the cells and, thus, block the process of carcinogenesis from happening [12]. Furthermore, the researchers found that ApobrdU positive cells indicated Nano Cur favorably stimulated apoptosis in tumor cells over normal cells.

\section{Conclusion}

Curcumin encapsulated in a range of drug devices can act as a therapeutic agent against various cancer sites, but there are many problems and limitations with previously mentioned delivery systems for curcumin that hinders its use in prevention and treatments of several cancer sites: 1) Moderate or limited loading capacity of many delivery devices, for example cyclodextrin in polymeric delivery system for curcumin, 2) Hydrophobic nature of curcumin results in a very low solubility in aqueous solutions and, hence, poor bioavailability especially upon oral administration of this pharmacological active curcumin, 3) Stability of curcumin delivery systems is another challenge that limits its use in cancer chemotherapy, and 4) Multidrug resistance is a major obstacle upon using chemotherapy against cancers.

\section{References}

[1] Sankaranarayanan, R. and Ferlay, J. (2006) Worldwide Burden of Gynaecological Cancer: The Size of the Problem. Best Practice \& Research Clinical Obstetrics \& Gynaecology, 20, 207-225. http://dx.doi.org/10.1016/j.bpobgyn.2005.10.007

[2] Siegel, R., Ma, J., Zou, Z. and Jemal, A. (2014) Cancer Statistics, 2014. CA: A Cancer Journal for Clinicians, 64, 9-29. http://dx.doi.org/10.3322/caac.21208

[3] Jemal, A., Bray, F., Center, M.M., Ferlay, J., Ward, E. and Forman, D. (2011) Global Cancer Statistics. CA: A Cancer Journal for Clinicians, 61, 69-90. http://dx.doi.org/10.3322/caac.20107

[4] Zheng, L.D., Tong, Q.S. and Wu, C.H. (2004) Growth-Inhibitory Effects of Curcumin on Ovary Cancer Cells and Its Mechanisms. Journal of Huazhong University of Science and Technology, 24, 55-58. http://dx.doi.org/10.1007/BF02830706

[5] Zheng, L.D., Tong, Q.S. and Wu, C.H. (2006) Growth Inhibition and Apoptosis Inducing Mechanisms of Curcumin on Human Ovarian Cancer Cell Line A2780. Chinese Journal of Integrative Medicine, 12, 126-131.

http://dx.doi.org/10.1007/BF02857359 
[6] Cai, Q., Shi, M., Yao, L., Mao, Y., Ming, Y. and Ouyang, G. (2006) Antiproliferation and Apoptosis Induced by Curcumin in Human Ovarian Cancer Cells. Cell Biology International, 30, 221-226.

[7] Sun, M., Su, X., Ding, B., He, X., Liu, X., Yu, A., Lou, H. and Zhai, G. (2012) Advances in Nanotechnology-Based Delivery Systems for Curcumin. Nanomedicine, 7, 1085-1100. http://dx.doi.org/10.2217/nnm.12.80

[8] Sharma, R.A., Gescher, A.J. and Steward, W.P. (2005) Curcumin: The Story So Far. European Journal of Cancer, 41, 1955-1968. http://dx.doi.org/10.1016/j.ejca.2005.05.009

[9] Ghosh, D., Choudhury, S.T., Ghosh, S., Mandal, A.K., Sarkar, S., Ghosh, A., Saha, K.D. and Das, N. (2012) Nanocapsulated Curcumin: Oral Chemopreventive Formulation against Diethylnitrosamine Induced Hepatocellular Carcinoma in Rat. Chemico-Biological Interactions, 195, 206-214. http://dx.doi.org/10.1016/j.cbi.2011.12.004

[10] Chen, Y., Wu, Q., Zhang, Z., Yuan, L., Liu, X. and Zhou, L. (2012) Preparation of Curcumin-Loaded Liposomes and Evaluation of Their Skin Permeation and Pharmacodynamics. Molecules, 17, 5972-5987. http://dx.doi.org/10.3390/molecules17055972

[11] Dhule, S.S., Penfornis, P., Frazier, T., Walker, R., Feldman, J., Tan, G., He, J., Alb, A., John, V., EngSca, D. and Pochampally, R. (2012) Curcumin-Loaded $\gamma$-Cyclodextrin Liposomal Nanoparticles as Delivery Vehicles for Osteosarcoma. Nanomedicine: Nanotechnology, Biology, and Medicine, 8, 440-451.

[12] Li, L., Braiteh, F.S. and Kurzrock, R. (2005) Liposome-Encapsulated Curcumin: In Vitro and in Vivo Effects on Proliferation, Apoptosis, Signaling, and Angiogenesis. Cancer, 104, 1323-1331.

[13] Huang, M.T., Lu, Y.P., Chang, R.L., Fisher, C., Manchand, P.S., Newmark, H.L. and Conney, A.H. (1995) Effects of Curcumin, Demethoxycurcumin, Bisdemethoxycurcumin and Tetrahydrocurcumin on 12-0-Tetradecanoylphorbol-13Acetateinduced Tumor Promotion. Carcinogenesis, 16, 2493-2497.

[14] Raheem, F.W. and Raheem, M.A. (2008) Gynecological Cancer Incidence in a Hospital Population in Saudi Arabia: The Effect of Foreign Immigration over Two Decades. Journal of Obstetrics and Gynaecology Research, 34, 538-542. http://dx.doi.org/10.1111/j.1447-0756.2008.00735.x

[15] Ganta, S. and Amiji, M. (2009) Coadministration of Paclitaxel and Curcumin in Nanoemulsion Formulations to Overcome Multidrug Resistance in Tumor Cells. Molecular Pharmaceutics, 6, 928-939. http://dx.doi.org/10.1021/mp800240j

[16] Kumar, S.S.D., Surianarayanan, M., Vijayaraghavan, R., Mandal, A.B., MacFarlane, D.R. and Veena, M.S. (2014) Curcumin Loaded Poly(2-hydroxyethyl methacrylate) Nanoparticles from Gelled Ionic Liquid-In Vitro Cytotoxicity and Anti-Cancer Activity in SKOV-3 Cells. European Journal of Pharmaceutical Sciences, 51, 34-44. http://dx.doi.org/10.1016/j.ejps.2013.08.036

[17] Crist, K.A., Zhang, Z., You, M., Gunning, W.T., Conran, P.B., Steele, V.E. and Lubet, R.A. (2005) Characterization of Rat Ovarian Adenocarcinomas Developed in Response to Direct Instillation of 7,12-Dimethylbenz[ $\alpha]$ Anthracene (DMBA) Coated Suture. Carcinogenesis, 26, 951-957. http://dx.doi.org/10.1093/carcin/bgi039

[18] Sait, K. (2011) Conservative Treatment of Ovarian Cancer Safety, Ovarian Function Preservation, Reproductive Ability, and Emotional Attitude of the Patients in Saudi Arabia. Saudi Medical Journal, 32, 913-918.

[19] Wahl, H.L.T., Griffith, K., Choi, M. and Liu, J. (2007) Curcumin Enhances Apo2L/TRAIL-Induced Apoptosis in Chemoresistant Ovarian Cancer Cells. Gynecologic Oncology, 105, 104-112. http://dx.doi.org/10.1016/j.ygyno.2006.10.050

[20] Yunos, N.M., Beale, P., Yu, J.Q. and Hug, F. (2011) Synergism from Sequenced Combinations of Curcumin and Epigallocatechin-3-gallate with Cisplatin in the Killing of Human Ovarian Cancer Cells. Anticancer Research, 31, 11311140 .

[21] Selvendiran, K., Ahmed, S., Dayton, A., Kuppusamy, M.L., Rivera, B.K., Kálai, T., Hideg, K. and Kuppusamy, P. (2011) HO-3867, A Curcumin Analog, Sensitizes Cisplatin-Resistant Ovarian Carcinoma, Leading to Therapeutic Synergy through STAT3 Inhibition. Cancer Biology and Therapy, 12, 837-845. http://dx.doi.org/10.4161/cbt.12.9.17713

[22] AL-Badawi, I.A., Brasher, P.M., Ghatage, P., Nation, J.G., Schepansky, A. and Stuart, G.C. (2002) Postoperative Chemotherapy in Advanced Ovarian Granulosa Cell Tumors. International Journal of Gynecological Cancer, 12, 119123. http://dx.doi.org/10.1046/j.1525-1438.2002.01067.x

[23] Stirling, G., Khalil, A.M., Nada, G.N., Saad, A.A. and Raheem, M.A. (1979) Malignant Neoplasms in Saudi Arabia. Cancer, 44, 1543-1548. http://dx.doi.org/10.1002/1097-0142(197910)44:4<1543::AID-CNCR2820440456>3.0.CO;2-N

[24] Al-Badawi, I.A., AlSubhi, J., Salem, H., Abduljabbar, A., Balaraj, K. and Munkarah, A. (2010) Laparoscopic Ovarian Transposition before Pelvic Irradiation. International Journal of Gynecological Cancer, 20, 1082-1086. http://dx.doi.org/10.1111/IGC.0b013e3181e2ace5

[25] Kakkar, V., Singh, S., Singla, D. and Kaur, I.P. (2011) Exploring Solid Lipid Nanoparticles to Enhance the Oral Bio- 
availability of Curcumin. Molecular Nutrition \& Food Research, 55, 495-503. http://dx.doi.org/10.1002/mnfr.201000310

[26] Al-Badawi, I.A., Munkarah, A.R., Tulbah, A., Babic, I.I., Al Husaini, H. and Ahmad, S. (2013) A Detailed Study of Patients and Tumor Characteristics of Epithelial Ovarian Cancer in Saudi Women. International Journal of Gynecological Cancer, 23, 456-460. http://dx.doi.org/10.1097/IGC.0b013e318284aafe

[27] Tamaddonfard, E., Hamzeh-Gooshchi, F. and Hamzeh-Gooshchi, N. (2010) Effect of Curcumin on Morphine-Induced Antinociception in Acute Corneal Pain in Rats. International Journal of Veterinary Research, 4, 127-131.

[28] LoTempio, M.M., Veena, M.S., Steele, H.L., Ramamurthy, B., Ramalingam, T.S., Cohen, A.N., Chakrabarti, R., Srivatsan, E.S. and Wang, M.B. (2005) Curcumin Suppresses Growth of Head and Neck Squamous Cell Carcinoma. Clinical Cancer Research, 11, 6994-7002. http://dx.doi.org/10.1158/1078-0432.CCR-05-0301

\section{List of Abbreviations}

\begin{tabular}{cc}
\hline Caspase-3 & Cysteinyl aspartate specific protease-3 \\
CCNGs & Curcumin loaded chitin nanogels \\
EMSA & Electrophoretic Mobility Shift Assay \\
MTT & 3-(4,5-dimethylthiazol-2-yl)-2,5-diphenyltetrazolium bromide \\
NF- ${ }_{\text {K }}$ & Nuclear factor-Kappa B \\
SKOV3 & Ovarian cancer drug resistant cells \\
PHEMA & Poly(2-hydroxyethyl methacrylate) nanoparticles \\
SKOV-3 & Sensitive human ovarian cancer cells \\
STAT3 & Signal transducer and activator of transcription-3 \\
\hline
\end{tabular}


Scientific Research Publishing (SCIRP) is one of the largest Open Access journal publishers. It is currently publishing more than 200 open access, online, peer-reviewed journals covering a wide range of academic disciplines. SCIRP serves the worldwide academic communities and contributes to the progress and application of science with its publication.

Other selected journals from SCIRP are listed as below. Submit your manuscript to us via either submit@scirp.org or Online Submission Portal.
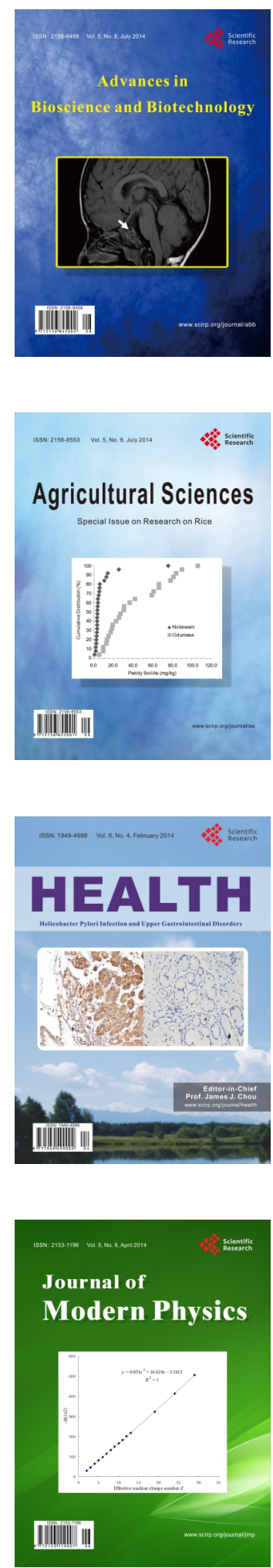
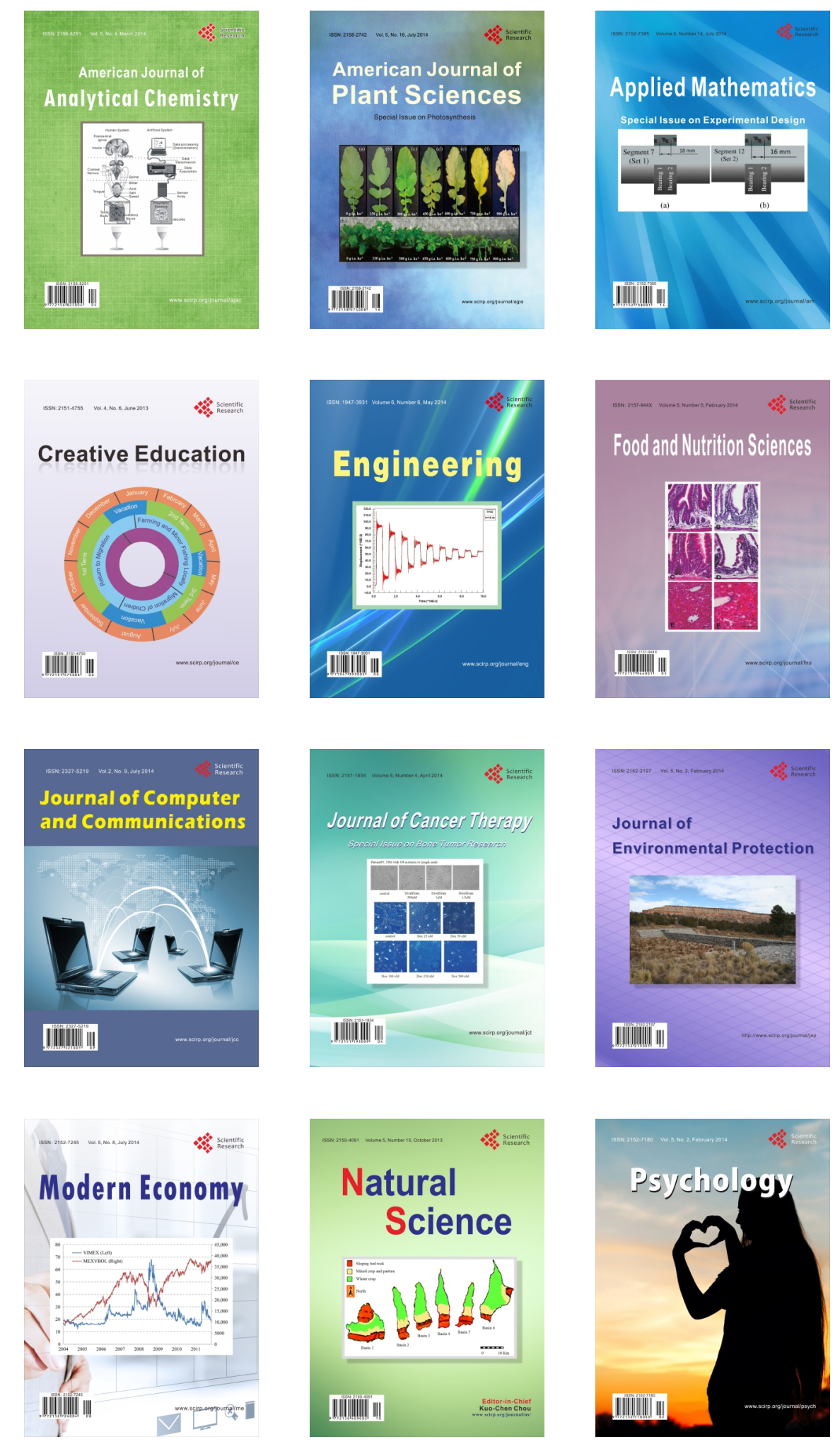